\title{
Correction to: Methods to adjust for multiple comparisons in the analysis and sample size calculation of randomised controlled trials with multiple primary outcomes
}

Victoria Vickerstaff ${ }^{1} 2^{*}$, Rumana Z. Omar ${ }^{2}$ and Gareth Ambler $^{2}$

Correction: BMC Med Res Methodol (2019) 19:129

https://doi.org/10.1186/s12874-019-0754-4

In the original publication of this article [1], "=1 - =1 $\approx \mathrm{s}$ " was mistakenly added after the sentence "In this method, the unadjusted $p$-values pj are multiplied by the number of primary outcome" in the Methods section, and should be deleted.

The original article has been corrected.

Published online: 22 July 2019

\section{Reference}

1. Vickerstaff $V$, et al. Methods to adjust for multiple comparisons in the analysis and sample size calculation of randomised controlled trials with multiple primary outcomes. BMC Med Res Methodol. 2019;19:129. https://doi.org/10.1186/s12874-019-0754-4.

\footnotetext{
* Correspondence: v.vickerstaff@ucl.ac.uk

${ }^{1}$ Marie Curie Palliative Care Research Department, Division of Psychiatry, University College London, Gower Street, London WC1E 6BT, UK

${ }^{2}$ Department of Statistical Science, University College London, Gower Street, London WC1E 6BT, UK
} 